\title{
¿Qué se entiende por ciudadanía global?
}

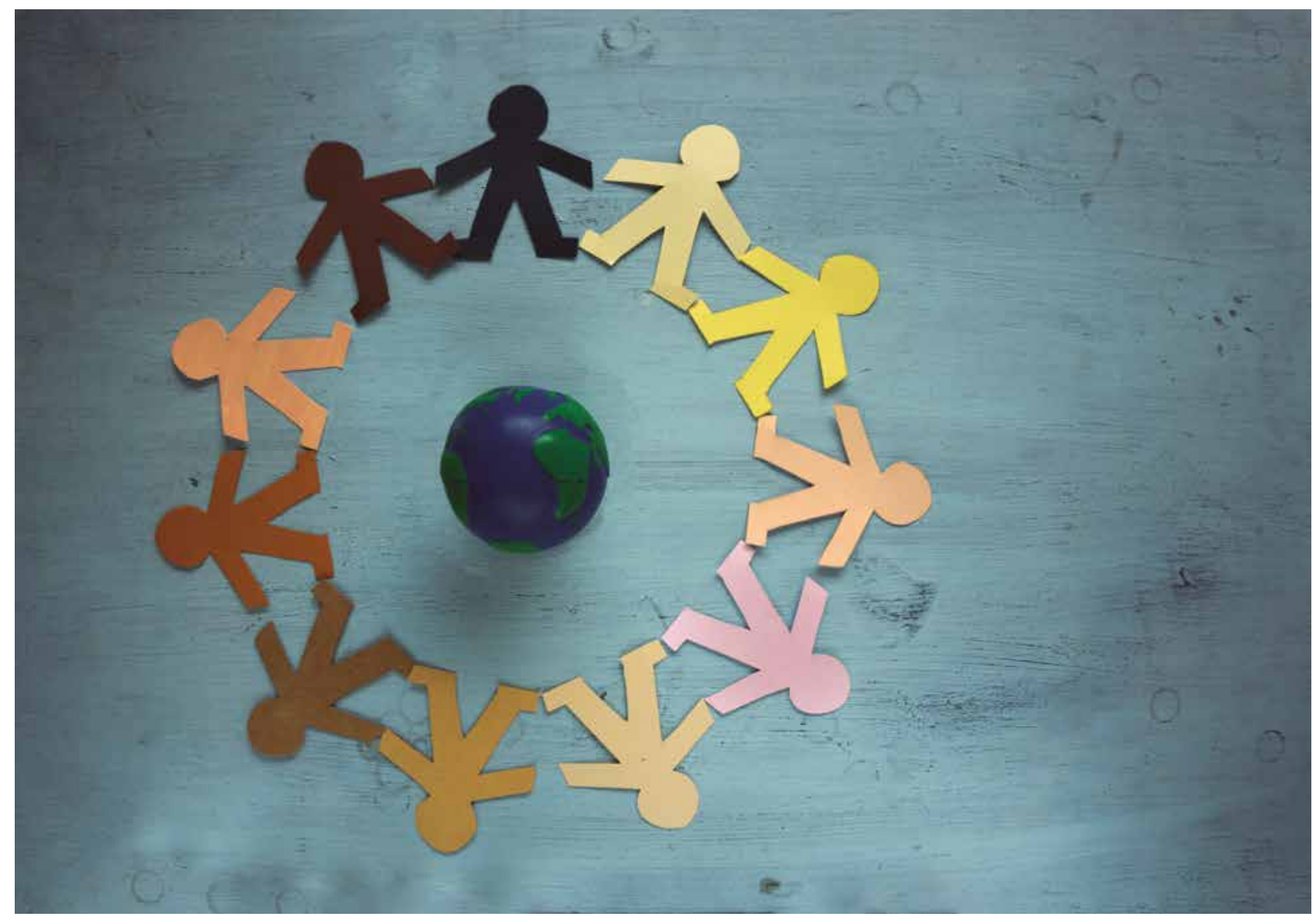

No hay un acuerdo entre los distintos organismos internacionales cuando se refieren a la ciudadanía global. Todos coinciden en su importancia y en la urgencia y necesidad de incorporarla a la vida escolar, pero es

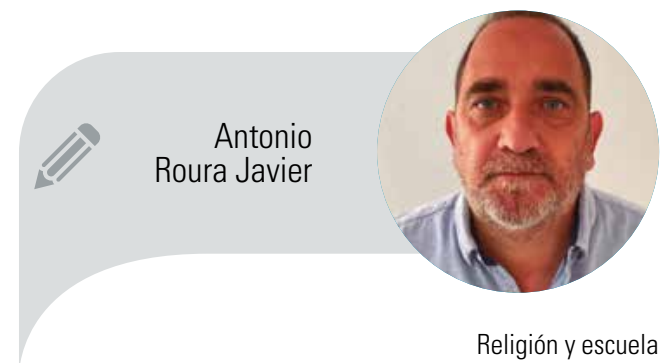
relevante conocer los diferentes enfoques —antropológicos, económicos, sociales y educativos- que quieren transmitir para no incorporarlos acríticamente a nuestras escuelas. 


\section{Un concepto con raíces seculares}

El término "ciudadanía global", especialmente usado por la OCDE, o "ciudadanía mundial", expresión utilizada por la Unesco, remite, y es necesario subrayarlo, a concepciones religiosas, filosóficas, morales y políticas con una tradición de siglos. Desde los estoicos a los filósofos modernos, los grandes pensadores y las tradiciones religiosas lo han utilizado con denominaciones diversas cuando han apelado y apelan a un universalismo catolicismo - que ha podido y puede correr el riesgo de caer en la insensibilidad frente a las culturas particulares, pero que ha tenido y tiene el valor de sentar las bases de derechos humanos inalienables, impulsar instituciones que los defiendan y promover la justicia en el mundo.

En el contexto actual de globalización, su uso se ha hecho especialmente intenso en el campo educativo. Aunque el origen de la expresión está ligado a la educación para el desarrollo, promovida por las ONG y trasladado a las aulas como una formación complementaria y extracurricular, en las dos décadas primeras del siglo XXI, la ciudadanía global (mundial) de la mano de las instituciones mundiales se viene incorporando a los currículos, la cultura escolar y las finalidades educativas de los sistemas educativos de los países.

\section{La ciudadanía global impulsada por las ON GD}

Las organizaciones no gubernamentales para el desarrollo (ONGD) comprometidas con la cooperación internacional han buscado incluir una perspectiva global en los currículos de las escuelas. Todos estos esfuerzos han estado enmarcados en lo que se ha llamado Educación para el desarrollo.

Podemos detectar cuatro dimensiones temporales que se han ido sucediendo en este tipo de educación: la primera pone el énfasis en lo caritativo asistencial, la segunda tiene un enfoque desarrollista, la tercera ofrece una visión crítica y solidaria del desarrollo, y la cuarta postula un modelo de desarrollo humano y sostenible (Oxfam-Reino

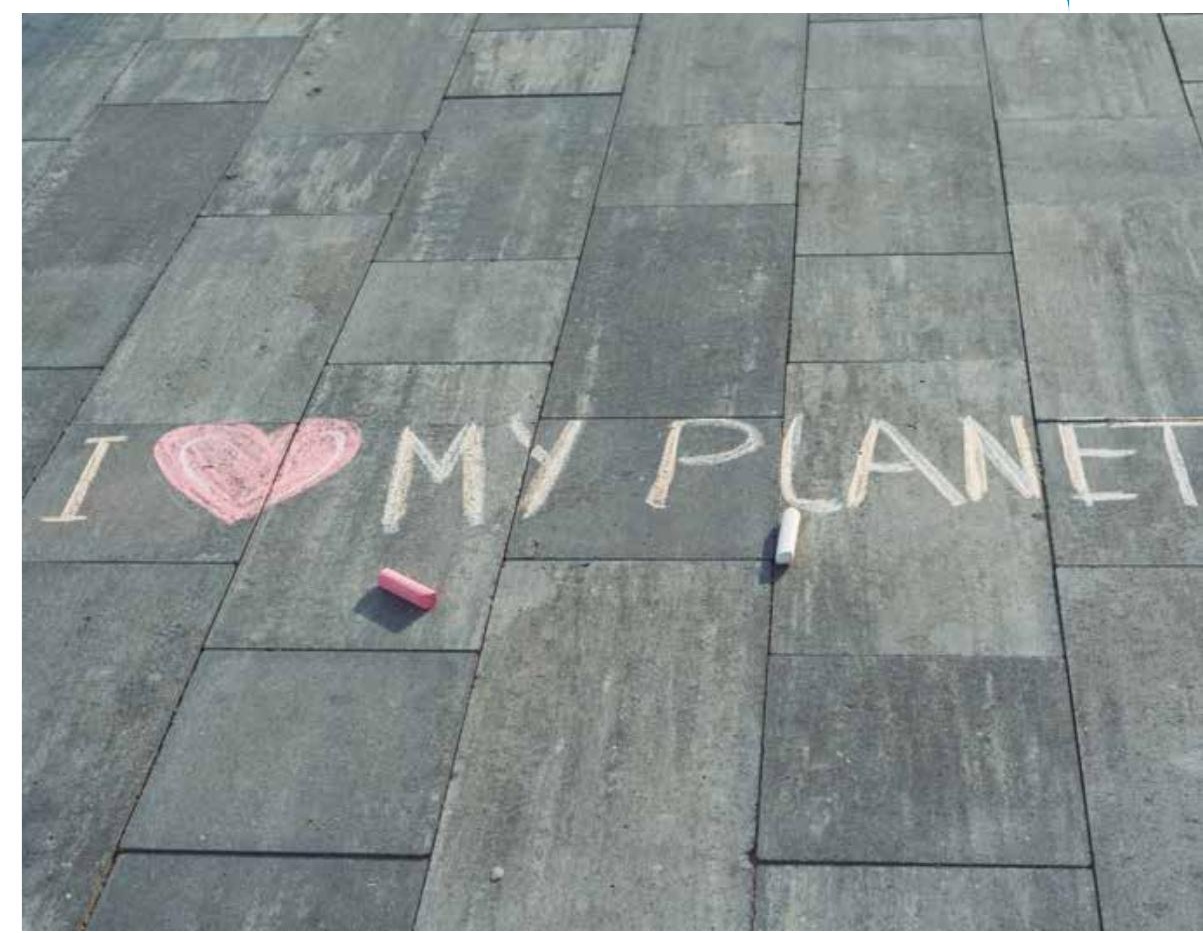

Unido, 2006; Intermón Oxfam, CIDAC, Inizjamed, UCODEP, 2007).

Bastantes ONGD están proponiendo en los últimos años una nueva perspectiva de esta Educación para el desarrollo. Consideran que hay que transformarla en lo que denominan una Educación para una Ciudadanía Global (EpCG). Esta nueva concepción permite una mejor comprensión de la globalización y sus efectos y se centra más en la formación ética y política de personas para que se comprometan en la transformación del mundo. Desde esta nueva perspectiva, un manifiesto internacional elaborado el año 2008 concibe la Educación para la Ciudadanía Global del siguiente modo:

\begin{abstract}
Entendemos por tal una educación que contribuye a la formación de ciudadanos y ciudadanas responsables, comprometidos con la justicia y la sostenibilidad del planeta, que promueve el respeto y la estima de la diversidad como fuente de enriquecimiento humano, la defensa del medio ambiente y el consumo responsable, el respeto de los derechos humanos individuales y colectivos, la igualdad de género, la valoración del diálogo como instrumento para la resolución pacífica de los conflictos, la participación, la corresponsabilidad y el compromiso en la construcción de una sociedad justa, equitativa y solidaria.
\end{abstract}

OXfam-Intermon, Cidac, INIJJAmed, UCOdep, 2008

¿En qué coordenadas se mueve esta ciudadanía global según estas ONGD?: 

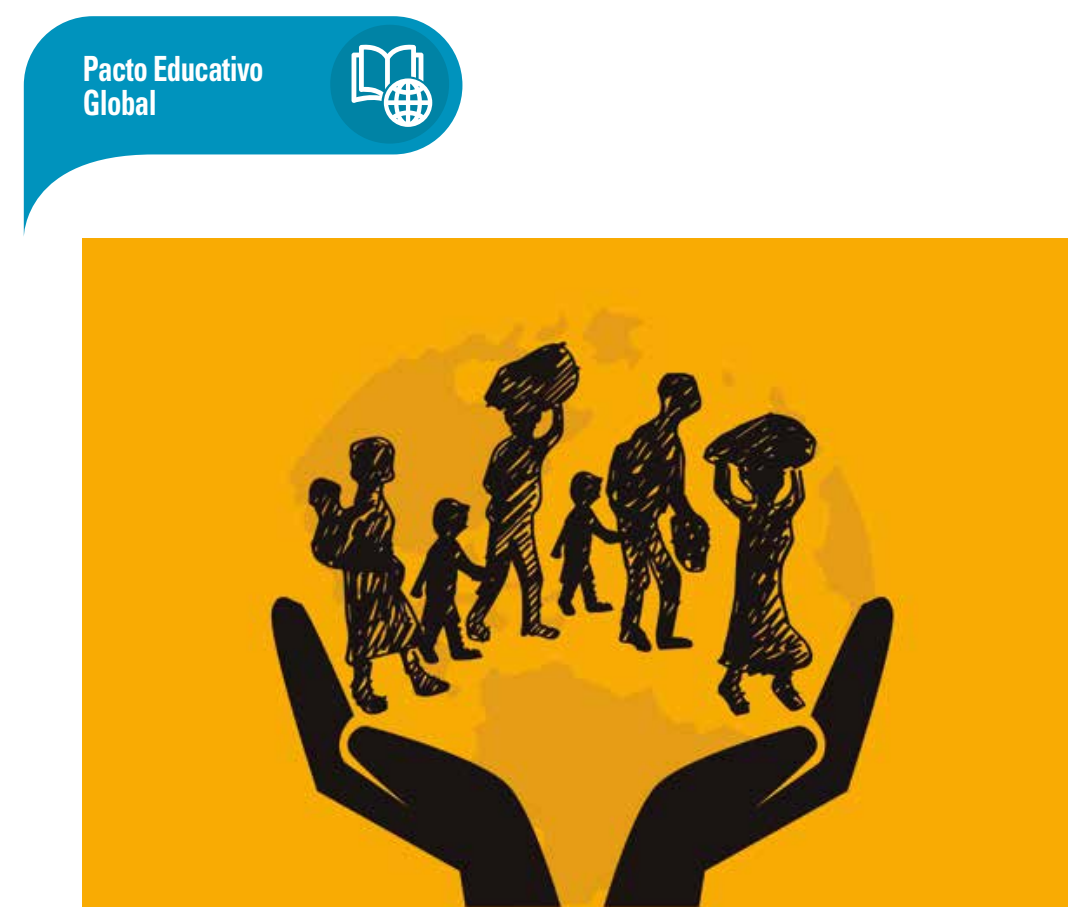

derechos humanos, responsabilidad ecológica ante la destrucción medioambiental de la Tierra, igualdad de género y valoración de la diversidad.

Énfasis distintos aparecen respecto a diversas nociones de "ciudadanía mundial": participación, compromiso, acción, defensa del cuidado del planeta, una mirada que integra el valor de lo local y lo particular evitando ópticas uniformadoras. En este sentido, se afirma que "La Educación para la Ciudadanía Global trata de desarrollar en los jóvenes una ciudadanía cosmopolita planetaria que rompa la lógica bipolar que contrapone la identidad universal a la particular, el 'nosotros' al 'vosotros' o 'lo nuestro' a lo 'vuestro'" (Intermón Oxfam, CIDAC, Inizjamed, UCODEP, 2007).

Las ONGD que comparten el enfoque de la educación para la ciudadanía global confluyen con redes mundiales de movimientos sociales en el Foro Social Mundial, cuya primera celebración tuvo lugar en la ciudad brasileña de Porto Alegre. Desde este foro se está realizando una fuerte crítica del actual modelo de globalización y se está impulsando una ciudadanía internacionalista para lograr entre ONGD y movimientos sociales que "otro mundo sea posible" (Díaz-Salazar, 2003).

\section{La promoción de la ciudadanía global en la ONU y en la Unesco}

La ONU viene promoviendo formalmente la educación para la ciudadanía mundial (en inglés Global Citizenship) desde hace años. En septiembre de 2012 , el entonces secretario general de la ONU, Ban Ki Moon, incluyó en la Global Educa- tion First Initiative el fomento de la conciencia de ser ciudadanos del mundo. Esta tarea de formar una ciudadanía global es una de las tres prioridades que Naciones Unidas plantea para el siglo XXI en el terreno de la educación (ONU, 2012).

La ONU y la Unesco acogen así una idea que llevaba, al menos, una década siendo reflexionada y discutida no solo en el mundo de las ONGD, sino también en el mundo académico (Andreotti, Barker, \& Newell-Jones, 2006). Además, esta preocupación no había sido nunca extraña en el paradigma educativo moderno. Ya Kant afirmó que "las bases de un plan de educación deben hacerse cosmopolitamente, teniendo en cuenta un mundo mejor, más humano" (Kant, 1991).

La Unesco propone la siguiente definición de ciudadanía global: "La ciudadanía mundial se refiere a un sentido de pertenencia a una comunidad más amplia y a una humanidad común. Hace hincapié en la interdependencia política, económica, social y cultural y en las interconexiones entre los niveles local, nacional y mundial" (Unesco, 2015).

La Unesco no entra en la discusión sobre el estatuto legal de esta ciudadanía o en la polémica de si esta "extiende" la ciudadanía tradicional definida en términos de Estado-nación o "compite" con ella. Aunque es consciente de esta discusión, cree que todas las perspectivas tienen en común este "sentido de pertenencia" a la que hace mención. Sitúa a la ciudadanía global en términos de identidad de la persona y su modo de estar en el mundo.

Definida así, un proyecto de educación que promueva la ciudadanía global busca ser un factor de transformación: "La educación para la ciudadanía mundial aspira a ser un factor de transformación, inculcando los conocimientos, las habilidades, los valores y las actitudes que los educandos necesitan para poder contribuir a un mundo más inclusivo, justo y pacífico" (Unesco, 2015).

Se trata de un proyecto de extensión de la educación para la ciudadanía hacia su necesaria dimensión mundial y como 
tal, busca desarrollar aprendizajes en tres dimensiones: cognitiva, socioemocional y conductual.

Los esfuerzos de la Secretaría General de la ONU y de la Unesco han ayudado a la inclusión de la educación para la ciudadanía global en distintos ámbitos internacionales. Uno de ellos es la Agenda 2030 de los Objetivos de Desarrollo Sostenible (ODS), aprobada e impulsada por la Asamblea General de la ONU en septiembre de 2015.

El cuarto de los diecisiete objetivos de la Agenda 2030 de los ODS se centra en "Garantizar una educación inclusiva, equitativa y de calidad y promover oportunidades de aprendizaje durante toda la vida para todos". Para la consecución de este objetivo se establecen una serie de metas entre las que se destaca la promoción de la ciudadanía mundial: "Para 2030, garantizar que todos los alumnos adquieran los conocimientos teóricos y prácticos necesarios para promover el desarrollo sostenible, entre otras cosas, mediante la educación para el desarrollo sostenible y la adopción de estilos de vida sostenibles, los derechos humanos, la igualdad entre los géneros, la promoción de una cultura de paz y no violencia, la ciudadanía mundial y la valoración de la diversidad cultural y de la contribución de la cultura al desarrollo sostenible, entre otros medios" (ONU, 2015).

\section{La propuesta de la Unión Europea} sobre educación para la ciudadanía

Es una constante en la Unión Europea tratar de fomentar en las escuelas el aprendizaje de los valores de fondo que la configuran. Desde 2006 existe un marco de referencia de la UE sobre competencias en el que destacan las competencias cívicas y sociales. Toda la legislación educativa de los países de la Unión Europea ha tenido que incorporarlas. Se conciben del siguiente modo:

Estas competencias incluyen las personales, interpersonales e interculturales y recogen todas las formas de comportamiento que preparan a las personas para participar de una manera eficaz y constructiva en la

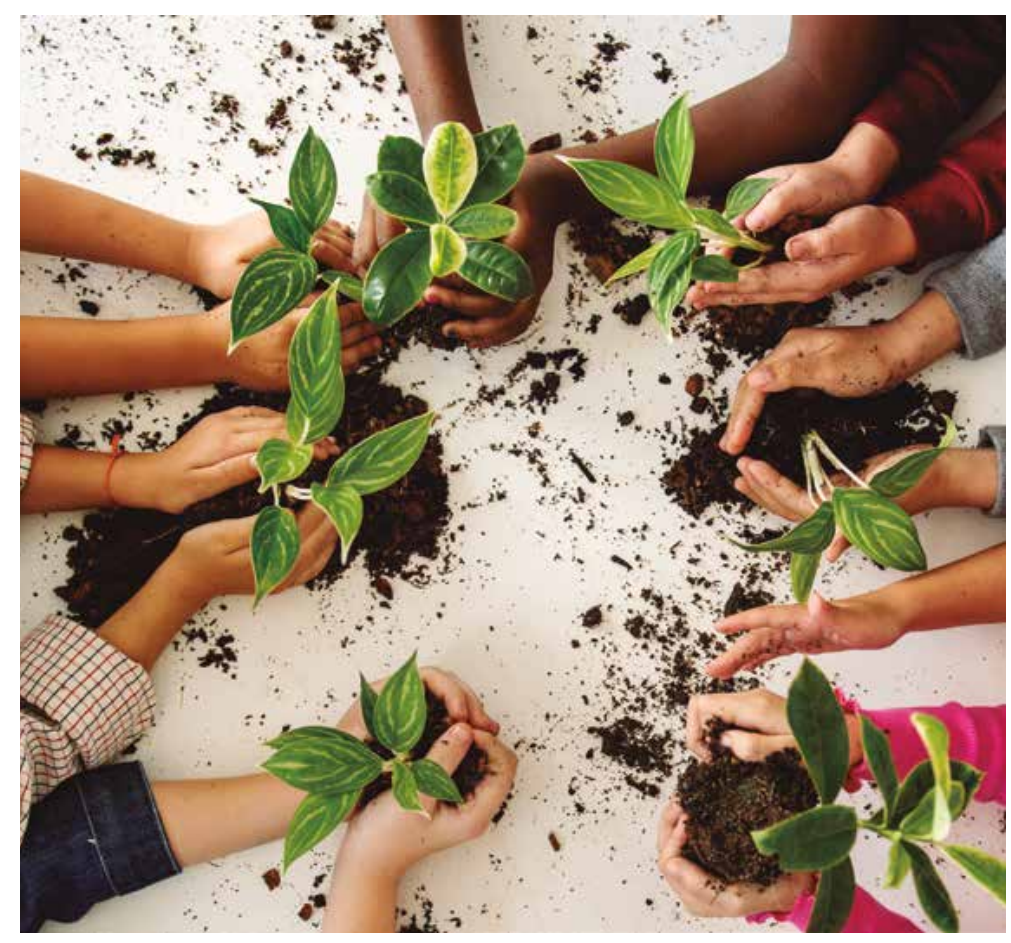

vida social y profesional, especialmente en sociedades cada vez más diversificadas, y, en su caso, para resolver conflictos. La competencia cívica prepara a las personas para participar plenamente en la vida cívica gracias al conocimiento de conceptos y estructuras sociales y políticas, y al compromiso de participación activa y democrática.

Parlamento y Consejo de la Unión Europea, 2006

En el Informe Eurydice sobre educación, publicado en 2017, se afirma que la educación en ciudadanía en Europa está concebida como una "área curricular para promover la convivencia armónica y favorecer el desarrollo de los individuos y de las comunidades en que viven". A diferencia de la educación cívica, que se circunscribe solo al conocimiento de la estructura constitucional del país y de sus instituciones políticas, esta materia de educación ciudadana ha de englobar competencias más generales, como la responsabilidad social y habilidades que aseguran un desarrollo personal (Comisión Europea/EACEA/Eurydice, 2017).

\section{La propuesta de la OCDE en el Informe PISA 2018}

La Organización para la Cooperación y el Desarrollo Económico (OCDE), que coordina la acción de los 35 Estados más ricos del mundo, incluye en su Informe del Programa Internacional para la EvaIuación de los Estudiantes (Informe PISA 


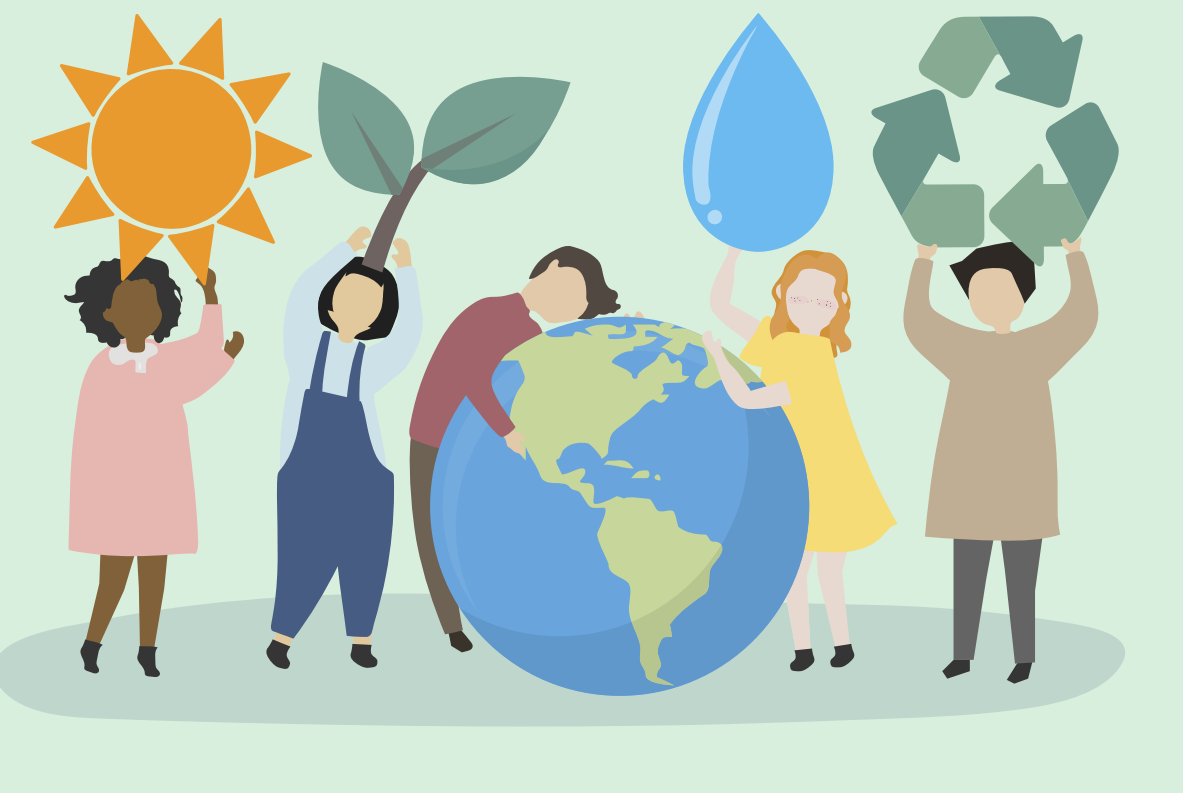

2018) la evaluación de lo que ha llamado competencia global. Así, a la evaluación que desde el año 2000 se ha ido haciendo de las competencias de lectura, ciencias y matemáticas, se suma esta nueva competencia que se describe así:

La competencia global es una capacidad multidimensional. Individuos competentes globalmente pueden: examinar asuntos locales, globales e interculturales, comprender y apreciar diferentes perspectivas y miradas del mundo, interactuar exitosa y respetuosamente con otros, tomar acciones responsables para la sostenibilidad y el bienestar colectivo.

OCDE, 2018

Esta competencia incluye distintos componentes que a su vez serán evaluados de forma distinta: conocimientos, habilidades cognitivas, actitudes y habilidades sociales. Estas capacidades y la incorporación de esta competencia en general se han justificado como una manera de responder a cuatro necesidades:

7 Para vivir armoniosamente en sociedades multiculturales, en un contexto donde los conflictos étnicos y culturales son la fuente más importante de violencia política en el mundo.

- Para prosperar en un mercado laboral cambiante, en un contexto donde los empleadores buscan a quien más fácilmente se adapte y sea capaz de aplicar y transferir sus habilidades y conocimientos a nuevos entornos.

7 Para usar las plataformas de medios de comunicación de manera efectiva y responsable, en un contexto en el que los radicales avances en las tecnologías digitales han transformado las perspectivas de los jóvenes en el mundo, sus interacciones y su percepción de sí mismos.

त Para apoyar las metas de desarrollo sostenible, en un contexto donde se requiere del compromiso de las nuevas generaciones para responder a los desafíos sociales y ambientales (OCDE, 2018).

La OCDE acoge así la propuesta de la ONU y la Unesco. Y lo hace a su modo: primero, no utilizando el concepto "ciudadanía", evitando así un término que está aún en construcción y que contiene una dimensión política; y segundo, incorporando la idea de la empleabilidad y la adaptabilidad del alumnado en el mundo global. Ahora bien, la OCDE opta junto a la ONU por apoyar una educación que sirva no solo a los intereses particulares, sino al bien universal. Se sostiene en dos grandes valores: el valor de la diversidad cultural y, en el horizonte y como límite a esta diversidad, el valor "inviolable de la dignidad humana". La incorporación de estos valores que se concretan en actitudes a evaluar es justificada por el bien de todos:

\begin{abstract}
Valorar la dignidad humana y valorar la diversidad cultural contribuye a la competencia global porque constituyen filtros críticos a través de los cuales las personas procesan información sobre otras culturas y deciden cómo relacionarse con los demás y el mundo. Las personas que cultivan estos valores se vuelven más conscientes de sí mismas y de su entorno, y están fuertemente motivadas para luchar contra la exclusión, la ignorancia, la violencia, la opresión y la guerra.
\end{abstract} OCDE, 2018

\section{No hay consenso internacional sobre ciudadanía global}

Después de analizar a grandes rasgos propuestas de organizaciones e instituciones internacionales sobre ciudadanía glo- 
bal, podríamos tener la sensación de que por caminos diferentes e independientes se ha llegado a un acuerdo estable sobre los conceptos, las habilidades y las actitudes con las que configurar las escuelas y evaluar el grado de adquisición de esta competencia global. Pero no es así.

No debemos confundirnos y creer que hay un consenso internacional sobre el tema. En cada una de las propuestas presentadas hay un modelo político e ideológico sobre qué es el ser humano, cuál ha de ser su relación con la naturaleza, con los demás, con la riqueza, con los empobrecidos, con las minorías, etc. Algunos de estos modelos pueden estar conectados con sistemas económicos que utilizan la educación como un medio de reproducción social y como un instrumento para reajustar el rendimiento competencial de cada país.

No sería prudente adoptar en las escuelas cualquier desarrollo educativo del concepto de ciudadanía global sin valorar críticamente qué paradigmas estamos incorporando y enseñando a los estudiantes. Por ello, considero que hay que tener muy presente este pensamiento del papa Francisco expresado en el n. ${ }^{\circ} 214$ de la encíclica Laudato si':

La educación será ineficaz y sus esfuerzos serán estériles si no procura también difundir un nuevo paradigma acerca del ser humano, la vida, la sociedad y la relación con la naturaleza. De otro modo, seguirá avanzando el paradigma consumista que se transmite por los medios de comunicación y a través de los eficaces engranajes del mercado.

Papa Francisco, 2015

\section{HEMOS HABLADO DE}

\section{Ciudadanía global; ciudadanía mundial; derechos humanos; organismos internacionales; desarrollo.}

Este artículo fue solicitado por PADRES Y MAESTROS en marzo de 2021, revisado y aceptado en mayo de 2021.

\section{(®a) DARR SABER MÁS}

Andreotti, V., Barker, L., \& Newell-Jones, K. (2006). Critical Literacy in Global Citizenship Education. Centre for the Study of Social and Global Justice, University of Nottingham https://www.academia.edu/194048/Critical_Literacy_in_Global_Citizenship_Education_2006_

Comisión EUROPEA/EACEA/Eurydice. (2017). La educación para la ciudadanía en el contexto escolar europeo 2017. Informe de Eurydice. Luxemburgo: Oficina de Publicaciones de la Unión Europea. https://eacea.ec.europa.eu/national-policies/eurydice/content/ citizenship-education-school-europe-\%E2 \%80 \%93-2017_en]

Díaz-Salazar, R. (ed.). (2003). Justicia global. Las alternativas de los movimientos del Foro de Porto Alegre. (2. ${ }^{a}$ ed.). Barcelona: Icaria.

Díaz Salazar, R. (coord.). (2020). Ciudadanía global en el siglo XXI. Educar para que otro mundo sea posible. Madrid: SM.

Intermón OXfam, CIDAC, InIZJAmed, UCODEP. (2007). La Educación para una Ciudadanía Global en la Escuela de hoy. Documento de posicionamiento. https://web.oxfamintermon.org/sites/default/ files/documentos/files/091224_posicionamiento_es.pdf

INTERMÓn OXfam, CIDAC, InIZJAmEd, UCODEP. (2008). Educar para una ciudadanía global. Manifiesto Internacional. Madrid: Oxfam-Intermon http://www.kaidara.org/recursos/manifiesto-internacional-ecg/

Kant, I. (1991). Pedagogía. Madrid: Akal.

OCDE. (2018). Preparing our youth for an inclusive and sustainable world. The OECD PISA global competence framework. París: OCDE https://www.oecd.org/education/Global-competency-for-an-inclusiveworld.pdf

OCDE. (2019). Study on Social and Emotional Skills - SSES. http:/I www.oecd.org/education/ceri/social-emotional-skills-study/

ONU. (2012). Global Education First Initiative. New York: ONU http:// www.unesco.org/new/en/gefi/home/?

ONU. (2015). Objetivos de Desarrollo Sostenible. Nueva York: Publicaciones de la ONU https://www.un.org/sustainabledevelopment/es/

OXFAM - Reino Unido. (2006). Teaching Controversial Issues. A guide for teachers. London: Oxfam. https://www.oxfam.org.uk/education/resources/teaching-controversial-issues

Papa Francisco. (2015). Laudato si'. Sobre el cuidado de la casa común. Bilbao: Mensajero.

Parlamento Europeo y Consejo de la Unión Europea. (2006). Recomendación del Parlamento Europeo y del Consejo, de 18 de diciembre de 2006, sobre las competencias clave para el aprendizaje permanente. https://eur-lex.europa.eu/legal-content/ES/TXT/PDF/?uri $=$ CELEX:32006H0962\&from $=$ EN

Unesco. (2015). Educación para la ciudadanía mundial. Temas y objetivos de aprendizaje http://unesdoc.unesco.org/ images/0023/002338/233876S.pdf

VAN DAMmEn, D. (2019). La OCDE aspira a que el Informe PISA también mida educación social y emocional. https://www.lavanguardia.com/vida/20190403/461443907721/la-ocde-aspira-a-queinforme-pisa-tambien-mida-educacion-social-y-emocional.html 